Title: Vitamin D supplementation

Author: Laura Meyer

Date: March 30, 2010

Key words: Vitamin D, supplementation, overall mortality

Abstract: Vitamin D supplementation is an inexpensive intervention that has many known benefits, including the maintenance of bone health. Given the current interest in the potential benefits of adequate vitamin D status, many patients are supplementing. A meta-analysis published in the Archives of Internal Medicine in 2007 indicates that vitamin D supplementation is not associated with adverse effects; in fact, there appears to be an associated decrease in all-cause mortality.

This document was created by a medical student enrolled in the Primary Care Clerkship at the University of Minnesota Medical School as part of the course project. The aim of the project is to present information on a medical topic in the format of a patient education handout. It does not necessarily reflect the views of the University of Minnesota Medical School physicians and faculty. These materials are provided for informational purposes only and are in no way intended to take the place of the advice and recommendations of your personal health care provider. The information provided may no longer be up to date since it has not been reviewed since the date of creation. The information provided should not be used to diagnose a health problem or disease, or as a means of determining treatment. In the event of a medical emergency, immediately contact a doctor or call 911. 


\section{Vitamin D Supplementation}

Vitamin D performs many important roles in our bodies.

- Works with calcium to maintain strong bones

- May help the body use insulin properly

- May help decrease the growth of cells and blood vessels to protect against cancer

- May protect against high blood pressure

- May protect against autoimmune diseases

Sources of vitamin D

- The sun: humans make vitamin $\mathrm{D}$ when the skin is exposed to ultraviolet- $\mathrm{B}$ rays from the sun. People living in northern climates may not be in the sun enough to make adequate vitamin $\mathrm{D}$.

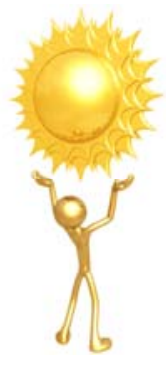

- Foods such as fish and cod liver oil

- Also added to foods such as fortified milk and eggs

- Supplements: either D2 or D3 400-2000 IU. The current recommendations by the U.S.
Institute of Medicine of the National Academies are 2000 IU for all individuals under 50,400 IU for individuals 5070 years old, and $600 \mathrm{IU}$ for those over 70. The Institute of Medicine is currently conducting a 24-month study on the adequacy and harmful effects of excess intake and will be relating their findings to chronic diseases. This consensus report should be available in summer 2010.

Safety: Vitamin D supplementation is considered safe when taken at the recommended dosages. Additionally, a large 2007 study indicated that vitamin $\mathrm{D}$ supplementation in the recommended dosages might be protective against death. Further research is needed to confirm these findings and to determine the underlying reasons for this.

For more information:

The U.S. Institute of Medicine of the National Academies: www.iom.edu

The Mayo Clinic: www.mayoclinic.com/health/vitam in-d/NS patient-vitamind 